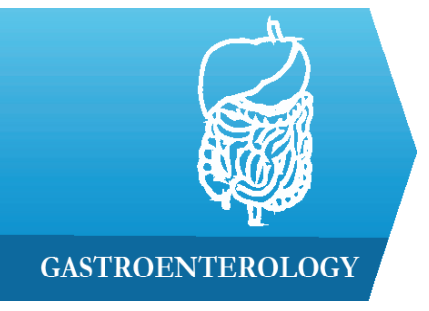

1) Gastroenterology Department, "Prof. Dr. Octavian Fodor" Institute of Gastroenterology and Hepatology, Cluj-Napoca, Romania

2) $2^{\text {nd }}$ Medical Department, Iuliu Hatieganu University of Medicine and Pharmacy, Cluj-Napoca, Romania
DOI: $10.15386 / \mathrm{mpr}-1323$

Manuscript received: 26.03.2019

Accepted: 13.06.2019

Address for correspondence:

ddumitrascu@umfcluj.ro

\title{
The management of hiatal hernia: an update on diagnosis and treatment
}

\author{
Alice Sfara $^{1}$, Dan L. Dumitrascu ${ }^{2}$
}

\begin{abstract}
Background and aim. Hiatal hernia $(\mathrm{HH})$ occurs quite frequently in the general population and is characterized by a wide range of non-specific symptoms, most of them related to gastroesophageal reflux disease. Treatment can be challenging at times, depending on the existence of complications. The most recent guideline regarding the management of hiatal hernia was released by the Society of American Gastrointestinal and Endoscopic Surgeons (SAGES) in the year 2013. This review aims to present the most recent updates on the diagnosis and management of hiatal hernia for clinical practitioners.
\end{abstract}

Methods. The PubMed database was screened for publications using the terms: "hiatal hernia", "paraesophageal hernia", "management", "treatment", "hiatal repair". A literature review of contemporary and latest studies was completed. The studies that we looked into include prospective, randomized trials, systematic reviews, clinical reviews and original articles. The information was compiled in narrative review format.

Results. This narrative review presents new data on the diagnosis and management of hiatal hernia. While the diagnostic pathway has remained virtually unchanged, new data have come to light regarding the surgical treatment of hiatal hernia. We present the imaging methods used for its diagnosis, as well as the medical and surgical treatment currently available.

Conclusion. In the last five years, there has been vast research in the field of hiatal hernia management, especially regarding the surgical treatment. However, unanswered questions still remain and solid updates on the guidelines have yet to be formulated. To address this, more randomized studies need to be done on subsets of patients, stratified by age, gender, symptoms and comorbidities.

Keywords: hiatal hernia, paraesophageal hernia, hiatal repair, management, treatment

\section{Introduction}

Hiatal hernia $(\mathrm{HH})$ represents a relatively frequent condition in the general population. It is caused by increased intra-abdominal pressure, which leads to the protrusion of the stomach and other abdominal viscera into the mediastinum $[1,2]$. Being overweight and elderly are the key risk factors in its development $[3,4,5]$. Other known risk factors include: multiple pregnancies, history of esophageal surgery, partial or full gastrectomy and certain disorders of the skeletal system associated with bone decalcification and degeneration $[3,6]$.

The incidence of symptomatic cases of hiatal hernia appears to be linked to the diagnosis of gastroesophageal reflux disease (GERD), the 2 conditions being closely associated [7,8]. The most characteristic manifestation one will find in hiatal hernia is gastroesophageal reflux, manifested through regurgitation and heartburn, while less common symptoms include dysphagia, epigastric or chest pain and even chronic iron deficiency anemia $[5,9,10]$. Large hernias can present with dysphagia, early satiety or regurgitation [6].

Conventionally, hiatal hernia used to be classified as either sliding or paraesophageal. The current anatomic classification of hiatal hernias consists of four types.

- Type I or sliding hernias associated with symmetrical ascent of the stomach through the diaphragmatic crus. Type I hernias represent more than $90 \%$ of cases of hiatal hernia and are known 
for their frequent association with GERD [8,11]. They are also associated with more severe degrees of esophagitis and Barrett's esophagus [12].

- Type II hernias or pure paraesophageal hernias $(\mathrm{PEH})-$ a portion of the gastric fundus herniates through the diaphragmatic hiatus adjacent to the esophagus, while the gastroesophageal junction remains in its normal anatomic position.

- Type III hernias are a combination of types I and II, where both the fundus and the gastroesophageal junction herniate through the hiatus. The fundus is above the gastroesophageal junction.

- Type IV hernias consist of a structure other than the stomach herniating through the thoracic cavity (small bowel, colon, omentum, peritoneum or spleen).

Types II-IV are referred to as paraesophageal hernias (PEH); their main clinical importance is due to their potential for ischemia, obstruction or volvulus [5]. The anatomic classification of hiatal hernia is necessary especially regarding the treatment approach, as indications for the surgical method are quite different between sliding and paraesophageal hernias.

\section{Diagnosis of esophageal hiatal hernia}

According to the Society of American Gastrointestinal and Endoscopic Surgeons, only investigations that will have an impact on the clinical management of the patient should be performed [2]. The diagnosis of hiatal hernia can be rather challenging at times due to the shift in the anatomy of the esophagogastric junction during deglutition, respiration and movement. A complete history and physical exam is mandatory, as they may reveal symptoms that were not previously apparent.

Bariumswallowradiographygivesvaluableinformation about the size of the herniated stomach and the location of the gastroesophageal junction [13]. Most studies agree that barium swallow still remains essential in the diagnosis of hiatal hernia [14]. According to Siegal et al., hiatal hernias can be diagnosed by this method if the axial herniation is greater than $2 \mathrm{~cm}$ [15]. The authors also recommend video-esophagram, as it provides the additional benefit of examining bolus transit. Oleynikov et al. point out the advantage of detecting esophageal motility dysfunction, stenosis and stricture related to GERD through barium swallow radiography [1]. The method can also help in the diagnosis of short esophagus [2]. The main disadvantage consists of exposure to radiation. The risks associated with radiation exposure accumulate over time and are closely linked to the number of X-ray exams one has been subjected to during their lifetime (Table I).

Esophagogastroduodenoscopy (EGD) has the advantage of real-time analysis of the esophageal mucosa, the mucosa of the stomach and duodenum, unlike Barrium swallow radiography. It is able to detect erosive esophagitis, Barrett's esophagus, Cameron's ulcer and even lesions suspicious for malignancy (mandatory to be documented in case of future surgical approach) [13]. However, it is often unable to visualize and appreciate a large hiatal hernia, especially when it comes to the organo-axial rotation of the stomach [18]. Duranceau et al. describe the challenges of providing an accurate endoscopic description of massive hernias, thus it is important to also perform a Barium swallow X-ray in order to decribe the hernia in an exact manner [16]. Roman et al. point out that when performing endoscopy, one must keep in mind that excess air insufflation of the stomach may exaggerate the size of the hernia [5].

Esophageal manometry provides valuable information regarding the motility of the esophagus. It is considered that a separation between the crural diaphragm and lower esophageal sphincter of $2 \mathrm{~cm}$ or more is diagnostic for hiatal hernia [11]. According to Andolfi et al., esophageal manometry should be performed especially before undergoing surgery, as it can rule out achalasia or other motility disorders. Before performing fundoplication surgery it is also essential to verify the integrity of the peristaltis of the esophagus, which can be done using high resolution manometry (HRM) as it provides real time pressure recording $[3,5]$. However, placement of the manometry catheter can be rather difficult; Dallemagne et al. have reported that the method is completed in less than $50 \%$ of cases [17].

Table I. Current diagnostic methods for hiatal hernia.

\section{Diagnostic technique}

Barium swallow X-ray $[1,3,5,6,13,14,16,17]$

Endoscopy

$[1,3,5,6,13,14,16,17]$

Manometry
$[1,3,5,6,13,14,16,17]$
pH testing
$[1,5,13,16,17]$
C T

$[3,13,16,17]$

\section{Evaluation}

size, location of hernia, motility dysfunction, stenosis, stricture related to GERD, short esophagus diagnosis

analysis of esophageal mucosa, erosive esophagitis, Barrett's esophagus, malignancy, Cameron's ulcers, swallowing difficulty

integrity of esophageal peristaltis, motility disorders, achalasia

quantitative analysis of reflux episodes

gastric volvulus, perforation, pneumoperitoneum, pneumomediastinum

\section{Warnings}

contraindicated in pregnancy, barium or iodine hypersensitivity, exposure to radiation

air insufflation of the stomach may exaggerate hernia size, difficulty to assess massive hernias accurately

difficulty in placing manometry catheter

unable to exactly define the configuration of the hernia, exposure to radiation 
$p H$ testing is not essential in the diagnosis of a hiatal hernia, but it has proven helpful in providing a quantitative analysis of reflux episodes as it correlates $\mathrm{pH}$ level with the patient's complaints of reflux [1]. In Duranceau et al's study, it is considered the gold standard to document acid reflux exposure in the esophageal lumen [16].

Computer tomography (CT) is not routinely recommended, but remains useful in conferring additional information on location and type of hiatal hernia. Usually, it is discovered incidentally while performing $\mathrm{CT}$ scan for a different indication [5]. Dallemagne et al. recommend CT scan in order to rule out complications like perforation, pneumoperitoneum or pneumomediastinum [17]. In case of paraesophageal hernia, it is a helpful method in the assessment of gastric volvulus [5].

Oleynikov et al., Duranceau et al. and Andolfi et al. have found that for a preoperative evaluation of a patient, barium swallow X-ray, upper endoscopy and manometry are essential $[1,13,16]$. Moreover, Weitzendorfer et al consider that in order to have a reliable exclusion of hiatal hernia prior to treatment, all three investigations must be performed [19].

\section{Treatment of hiatal hernia Medical appproach}

When confronted with a symptomatic hiatal hernia, it is usually due to acid reflux. Therefore, the aim is to reduce the symptoms of gastroesophageal reflux disease (GERD) by addressing gastric acid secretion. Lifestyle modifications are the first line of management and include the following: weight loss, elevating the head of the bed by 8 inches during sleep, avoidance of meals 2-3 hours before bedtime, elimination of "trigger" foods such as chocolate, alcohol, caffeine, spicy foods, citrus, carbonated drinks [20,21]. According to the American College of Gastroenterology, an 8-week course of PPI is the therapy of choice for symptom relief in GERD, with no major differences in the efficacy between the different types of PPIs [22]. Twice-daily PPI therapy can be recommended for patients with an inadequate symptom response to once-daily PPI [20]. The current recommendation is to use the minimal dose of PPI that is sufficient to control symptoms [5]. Other alternatives include histamine 2 receptor antagonists and antacids. Patients presenting with moderate symptoms can use these treatments on demand, while those with persistent symptoms despite PPI treatment should use them as an add-on treatment.
As for paraesophageal hernias, they are a different matter at hand: the gastric fundus has migrated above the diaphragm and, therefore, they are at a high risk of obstruction. Most of the patients that develop symptoms from paraesophageal hernia will experience little or no relief with medication such as proton pump inhibitors, histamine receptor antagonists or antacids [1]. It is helpful to take such medication, as it eases the symptoms, but the definitive treatment for paraesophageal hernia remains surgery.

Prokinetic drugs are not recommended in guidelines neither as monotherapy nor as add-on treatment as there is no evidence supporting their efficacy in the treatment of hiatal hernia associated with GERD [20,23].

\section{Surgical appproach}

The current guideline for the surgical treatment of hiatal hernia was elaborated by the Society of American Gastrointestinal and Endoscopic Surgeons (SAGES) in the year 2013. Through our literature research we have found that indications for surgery remain the same: symptomatic patients with paraesophageal hernia $[1,17,24]$, especially those with obstructive symptoms and gastric volvulus, which require urgent surgery [21]. When sliding hernia and symptoms of GERD are present, surgical approach might be considered, especially in cases where regurgitation persists despite medical treatment with PPI [5]. However, Andolfi et al. have suggested in their study that even asymptomatic patients younger than 50 should be considered for surgery [13,25]. Recently, a study performed by Straatman et al. has taken into account octogenarians and their indication for surgical repair. The conclusion was that elective repair in symptomatic patients should be the preferred approach in the octogenarian group [26] (Table II).

The SAGES Guidelines strongly recommend not repairing type I hiatal hernia in the absence of reflux disease and symptoms; this recommendation remains valid to this day, as sustained by several authors $[5,15]$. These guidelines also recommend performing hiatal hernia repair combined with other types of bariatric surgery, such as sleeve gastrectomy and gastric bypass. Recently, Mahawar et al. have performed a study that found sleeve gastrectomy to be the best bariatric option for obese patients with $\mathrm{HH}$ and/or GERD. They have also demonstrated that sleeve gastrectomy and hiatal hernia repair can safely be performed simultaneously [27].

Table II. Current therapeutic approaches of hiatal hernia.

\begin{tabular}{l|l|l} 
Type of hiatal hernia & \multicolumn{1}{c|}{ First line } & \multicolumn{1}{c}{ Second line } \\
Type I (sliding) hernia & $\begin{array}{l}\text { PPI - once daily, 8 week course treatment } \\
\text { Inadequate symptom control: PPI - twice daily, 8 week } \\
\text { course treatment }\end{array}$ & $\begin{array}{l}\text { Laparoscopic fundoplication (Nissen or } \\
\text { Toupet) - especially in case of symptom } \\
\text { persistence }\end{array}$ \\
$\begin{array}{ll}\text { Types II, III, IV } \\
\text { (paraesophageal) hernias }\end{array}$ & $\begin{array}{l}\text { Laparoscopic fundoplication (Nissen or Toupet) - } \\
\text { definitive treatment }\end{array}$ & $\begin{array}{l}\text { PPI, histamine receptor antagonists, antacids } \\
\text { - for symptom relief }\end{array}$
\end{tabular}


With regards to the technical approach, hiatal hernia can be repaired either transabdominally or via a transthoracic approach, usually through the left chest. The current standard procedure is laparoscopic fundoplication for both sliding and paraesophageal hernia [28]. Usually, a Nissen fundoplication $\left(360^{\circ}\right)$ is performed after most hiatal hernia repairs, unless there is a preexisting esophageal dysmotility, in which case the Toupet fundoplication $\left(270^{\circ}\right)$ is preferred [15]. There has been recent evidence that a complete fundoplication might be more beneficial as it has a reduced abnormality in the $\mathrm{pH}$ profile and a stronger impact [3]. Laparoscopic surgery provides the advantages of a minimally invasive approach, which consist of: shorter hospital stays, faster time of recovery, reduced post-operative pain and reduced pulmonary complications $[1,28]$. There are, of course, certain disadvantages of laparoscopic surgery which include two-dimensional imaging, limited motion of laparoscopic instruments and poor ergonomics for surgeons [28].

The technical considerations have remained mostly the same, however, new data has been brought to light regarding the use of mesh during paraesophageal hernia repair. The current position of the SAGES Gudelines regarding this topic is uncertain; there is inadequate data on the long-term to formulate a recommendation either for or against the use of mesh repair [2]. Although controversy still revolves around paraesophageal hernia repair, the general opinion seems to favor the use of mesh reinforcement. Zaman et al. have found a decrease in recurrence after laparoscopic paraesophageal hernia repair and mesh reinforcement, with similar results in both syntethic and biologic mesh [24]. Regarding the use of synthetic mesh, concerns have arisen as it has been associated with the development of esophageal erosion, stricture, dysphagia, obstruction and esophageal stenosis [3,24,29]. Zhang et al., Huddy et al. and Tam et al. have all found a reduced rate of hernia recurrence after mesh reinforcement compared to primary suture repair at shortterm follow-up (up to 12 months) [30,31,32]. Moreover, Oelschlager et al have demonstrated that a lower recurrence rate is associated with short-term follow-up for biologic mesh reinforcement, while with long-term follow-up the benefit is lost [33]. As a result, the short-term benefit may justify the use of biologic mesh among surgeons, but, more studies will be needed in order to establish a clear recommendation as a guideline.

One topic that is not mentioned at all in the 2013 SAGES Guidelines is represented by the role of robotic surgery in hiatal hernia repair. The development of the DaVinci telemanipulation system might overcome some of the limitations of standard laparoscopic approach by providing increased visualization through three-dimensional and stereoscopic vision and optimized ergonomics [28,34]. Vasudevan et al. have found in their study that the robotic approach to paraesophageal repair is effective and safe, with low complication rates, even in patients of older age and risk of complications [28]. However, Gehrig et al. concluded that there is no significant advantage of the DaVinci system over the conventional laparoscopic technique [35]. Their study found no significant difference regarding the operating time, intraoperative and postoperative complications and hospital stay between the robotic assisted surgery group and the laparoscopic surgery group. Moreover, the issue of cost remains an important drawback related to robotic surgery. General recommendations cannot be made yet regarding this field, as there are no large randomized trials comparing efficacy, outcomes and cost effectiveness of both robotic and laparoscopic paraesophageal hernia repairs.

\section{Conclusions}

Updates on the current guidelines of hiatal hernia management have yet to be made, as additional studies regarding long-term follow-up and stratified subsets of patients need to be designed. However, until these are available, whether mesh reinforcement and robotic approach can be used remains at the surgeon's discretion.

\section{References}

1. Oleynikov D, Jolley JM. Paraesophageal hernia. Surg Clin North Am. 2015;95:555-565.

2. Kohn GP, Price RR, DeMeester SR, Zehetner J, Muensterer OJ, Awad Z, et al. Guidelines for the management of hiatal hernia. Surg Endosc. 2013;27:4409-4428.

3. Yu HX, Han CS, Xue JR, Han ZF, Xin H. Esophageal hiatal hernia: risk, diagnosis and management. Expert Rev Gastroenterol Hepatol. 2018;12:319-329.

4. Bashashati M, Sarosiek I, McCallum RW. Epidemiology and mechanisms of gastroesophageal reflux disease in the elderly: a perspective. Ann N Y Acad Sci. 2016;1380:230-234.

5. Roman S, Kahrilas PJ. The diagnosis and management of hiatus hernia. BMJ. 2014;349:g6154.

6. Siegal SR, Dolan JP, Hunter JG. Modern diagnosis and treatment of hiatal hernias. Langenbecks Arch Surg. 2017;402:1145-1151.

7. Argyrou A, Legaki E, Koutserimpas C, Gazouli M, Papaconstantinou I, Gkiokas G, et al. Risk factors for gastroesophageal reflux disease and analysis of genetic contributors. World J Clin Cases. 2018;6:176-182.

8. Sugimoto M, Uotani T, Ichikawa H, Andoh A, Furuta T. Gastroesophageal Reflux Disease in Time Covering Eradication for All Patients Infected with Helicobacter pylori in Japan. Digestion. 2016;93:24-31.

9. Stein J, Connor S, Virgin G, Ong DE, Pereyra L. Anemia and iron deficiency in gastrointestinal and liver conditions. World J Gastroenterol. 2016;22:7908-7925.

10. Patoulias D, Kalogirou M, Feidantsis T, Kallergis I, Patoulias I. Paraesophageal Hernia as a Cause of Chronic Asymptomatic Anemia in a 6 Years Old Boy; Case Report and Review of the Literature. Acta Medica (Hradec Kralove). 2017;60(2):76-81.

11. Philpott H, Sweis R. Hiatus Hernia as a Cause of Dysphagia. Curr Gastroenterol Rep. 2017;19:40. 
12. Menezes MA, Herbella FAM. Pathophysiology of Gastroesophageal Reflux Disease. World J Surg. 2017;41:1666-1671.

13. Andolfi C, Jalilvand A, Plana A, Fisichella PM. Surgical Treatment of Paraesophageal Hernias: A Review. J Laparoendosc Adv Surg Tech A. 2016;26:778-783.

14. Lebenthal A, Waterford SD, Fisichella PM. Treatment and controversies in paraesophageal hernia repair. Front Surg. 2015;2:13.

15. Siegal SR, Dolan JP, Hunter JG. Modern diagnosis and treatment of hiatal hernias. Langenbecks Arch Surg. 2017;402:1145-1151.

16. Duranceau A. Massive hiatal hernia: a review. Dis Esophagus. 2016;29:350-366.

17. Dallemagne B, Quero G, Lapergola A, Guerriero L, Fiorillo C, Perretta S. Treatment of giant paraesophageal hernia: pro laparoscopic approach. Hernia. 2018;22:909-919.

18. Collet D, Luc G, Chiche L. Management of large paraesophageal hiatal hernias. J Visc Surg. 2013;150:395-402.

19. Weitzendorfer M, Köhler G, Antoniou SA, Pallwein-Prettner L, Manzenreiter L, Schredl P, et al. Preoperative diagnosis of hiatal hernia: barium swallow X-ray, high-resolution manometry, or endoscopy ? Eur Surg. 2017;49:210-217.

20. Kahrilas PJ, Shaheen NJ, Vaezi MF, Hiltz SW, Black E, Modlin IM, et al. American Gastroenterological Association Medical Position Statement on the management of gastroesophageal reflux disease. Gastroenterology. 2008;135:1383-1391.

21. Katz PO, Gerson LB, Vela MF Guidelines for the Diagnosis and Management of Gastroesophageal Reflux Disease . Am J Gastroenterol. 2013;108:308-328.

22. Katz PO, Gerson LB, Vela MF. Guidelines for the diagnosis and management of gastroesophageal reflux disease. Am J Gastroenterol. 2013;108:308-328; quiz 329.

23. Roman S, Kahrilas PJ. Mechanisms of Barrett's oesophagus (clinical): LOS dysfunction, hiatal hernia, peristaltic defects. Best Pract Res Clin Gastroenterol. 2015;29:17-28.

24. Zaman JA, Lidor AO. The Optimal Approach to Symptomatic Paraesophageal Hernia Repair: Important Technical Considerations. Curr Gastroenterol Rep. 2016;18:53.
25. Poncet G, Robert M, Roman S, Boulez JC. Laparoscopic repair of large hiatal hernia without prosthetic reinforcement : late results and relevance of anterior gastropexy. J Gastrointest Surg. 2010;14:1910-1916.

26. Straatman J, Groen LCB, van der Wielen N, Jansma EP, Daams F, Cuesta MA, et al. Treatment of paraesophageal hiatal hernia in octogenarians: a systematic review and retrospective cohort study. Dis Esophagus. 2018;31(7).

27. Mahawar KK, Carr WR, Jennings N, Balupuri S, Small PK. Simultaneous sleeve gastrectomy and hiatus hernia repair: a systematic review. Obes Surg. 2015;25:159-166.

28. Vasudevan V, Reusche R, Nelson E, Kaza S. Robotic paraesophageal hernia repair: a single-center experience and systematic review. J Robot Surg. 2018;12:81-86.

29. Rochefort M, Wee JO. Management of the Difficult Hiatal Hernia. Thorac Surg Clin. 2018;28:533-539.

30. Zhang C, Liu D, Li F, Watson DI, Gao X, Koetje JH, et al. Systematic review and meta-analysis of laparoscopic mesh versus suture repair of hiatus hernia: objective and subjective outcomes. Surg Endosc. 2017;31:4913-4922.

31. Huddy JR, Markar SR, Ni MZ, Morino M, Targarona EM, Zaninotto $G$, et al. Laparoscopic repair of hiatus hernia: Does mesh type influence outcome? A meta-analysis and European survey study. Surg Endosc. 2016;30:5209-5221.

32. Tam V, Winger DG, Nason KS. A systematic review and meta-analysis of mesh vs suture cruroplasty in laparoscopic large hiatal hernia repair. Am J Surg. 2016;211:226-238.

33. Oelschlager BK, Pellegrini CA, Hunter JG, Brunt ML, Soper NJ, Sheppard BC, et al. Biologic prosthesis to prevent recurrence after laparoscopic paraesophageal hernia repair: long-term follow-up from a multicenter, prospective, randomized trial. J Am Coll Surg. 2011;213:461-468.

34. Morelli L, Guadagni S, Mariniello MD, Pisano R, D’Isidoro $\mathrm{C}$, Belluomini MA, et al. Robotic giant hiatal hernia repair: 3 year prospective evaluation and review of the literature. Int $\mathrm{J}$ Med Robot. 2015;11:1-7.

35. Gehrig T, Mehrabi A, Fischer L, Kenngott H, Hinz U, Gutt CN, et al. Robotic-assisted paraesophageal hernia repair - a casecontrol study. Langenbecks Arch Surg. 2013;398:691-696. 